\title{
The bronchiectasis severity index and FACED score for bronchiectasis
}

\author{
Wei-jie Guan, Rong-chang Chen and Nan-shan Zhong
}

Affiliation: State Key Laboratory of Respiratory Disease, National Clinical Research Center for Respiratory Disease, Guangzhou Institute of Respiratory Disease, First Affiliated Hospital of Guangzhou Medical University, Guangzhou Medical University, Guangzhou, China.

Correspondence: Rong-chang Chen, State Key Laboratory of Respiratory Disease, National Clinical Research Center for Respiratory Disease, Guangzhou Institute of Respiratory Disease, First Affiliated Hospital of Guangzhou Medical University, 151 Yanjiang Road, Guangzhou, Guangdong, China. E-mail: chenrcavip.163.com

-

@ERSpublications

The BSI and FACED scores have complementary roles in the assessment of the severity of bronchiectasis http://ow.ly/Ui7IB

Bronchiectasis is a chronic airway disease which is associated with cough, sputum production, haemoptysis and/or other distressing symptoms, resulting from various aetiologies [1-4]. The vicious cycle, which consists of airway infection, airway inflammation and structural damage, predominates in the pathogenesis and progression of bronchiectasis [5]. Despite the disappointingly limited number of effective treatment approaches and evidence-based management recommendations, it is imperative to evaluate disease severity meticulously so that better therapeutic outcomes can be achieved.

To date, bronchiectasis has received insufficient attention, which might be attributable to the erroneous perception globally that this is an "orphan disease" and that this disease could sometimes be readily managed with traditional medications such as antibiotics. Nonetheless, in Oriental countries such as China, patients with bronchiectasis (regardless of their concomitant diseases) have been frequently encountered in respiratory outpatient clinics. Furthermore, bronchiectasis per se has been indeed challenging to evaluate, since there has been a lack of simple, well-validated composite measure. Conventionally, forced expiratory volume in $1 \mathrm{~s}\left(\mathrm{FEV}_{1}\right)$ has been employed to represent the disease severity in previous reports [6, 7]. Chest high-resolution computed tomography (HRCT) scores, such as the modified Reiff score [8-10] and Bhalla score [11], have also been proposed as cardinal markers of disease severity. However, FEV1 did not effectively aid in clinical decision making and HRCT score had a poor correlation with lung function [9], which mandated the derivation of a novel scoring system(s) for assessment of the severity of bronchiectasis.

Through the efforts of an international collaboration, CHALmers et al. [12] introduced the bronchiectasis severity index (BSI), which consisted of HRCT score, FEV1, Medical Research Council dyspnoea score, bacterial colonisation (Pseudomonas aeruginosa or other pathogenic bacteria), prior hospital admission and exacerbations. They found that the BSI was a sensitive tool in predicting future risks of hospitalisation and mortality. Another independent research group, led by MARTiNEZ-GARCIA et al. [13], simultaneously established the FACED score, which comprises FEV1, age, P. aeruginosa colonisation, radiological extension and dyspnoea. Similarly, the FACED score effectively predicted mortality. Both scoring systems

Received: Oct 182015 | Accepted: Oct 212015

Support statement: This work was supported by the Changiiang Scholars and Innovative Research Team in University ITR0961; The National Key Technology R\&D Program of the 12th National 5-Year Development Plan 2012BAI05B01; the National Key Scientific and Technology Support Program Collaborative Innovation of Clinical Research for Chronic Obstructive Pulmonary Disease and Lung Cancer grant 2013BAI09B09 (to N. Zhong and R. Chen); National Natural Science Foundation grant 81400010; and 2014 Scientific Research Projects for Medical Doctors and Researchers from Overseas, Guangzhou Medical University grant 2014C21 (W. Guan). Funding information for this article has been deposited with FundRef.

Conflict of interest: Disclosures can be found alongside the online version of this article at erj.ersjournals.com

Copyright OERS 2016 
include FEV1, P. aeruginosa colonisation, HRCT score and dyspnoea: the shared elements. Nonetheless, the disparity in the remaining components would still contribute to the differences in how these two scores reflect disease severity.

So what is the relationship between the BSI and FACED scoring system? Should the BSI and FACED score be utilised alone or complementarily in clinical settings? In an editorial previously published in the European Respiratory Journal (ERJ), SALEH and HURST [14] commented that both scores predicted long-term mortality well and that FACED appeared to be simpler. Nonetheless, BSI provided information regarding annual risk and outcomes of hospitalisation. Recently, Minov et al. [15] have compared bronchiectasis severity assessed with the BSI and FACED scores. They have demonstrated that approximately $45 \%$ of patients had mild bronchiectasis, $37.8 \%$ had moderate bronchiectasis and $16-18 \%$ had severe bronchiectasis. Both scores yielded very similar patterns of distribution of disease severity. However, the results appeared to be problematic because of several potential underlying limitations: a small sample size $(n=37)$, a low proportion of never-smokers (48.7\%) and patients colonised with P. aeruginosa (8.1\%), and the lack of long-term follow-up. Although suffering from a relatively small sample size and monocentric study design, our research group did notice some of the inherent distinction between these two scoring system: the differential distribution of patients with different intervals of FEV1 and prior hospitalisations. Compared with the BSI, the distribution of FACED scores appeared to be more skewed towards mild bronchiectasis (unpublished data). In light of these limitations in study design, further studies that directly and systematically compare the usefulness of both scoring system in bronchiectasis are therefore urgently indicated.

Now we have an opportunity to address these concerns. In this issue of the ERJ, ELLIS et al. [16] have performed a meticulous analysis of the data derived from a 19-year follow-up at the Royal Brompton Hospital (London, UK). In this study, 74 bronchiectasis patients were followed-up for a median of 18.8 years. Because of the time course and number of deaths $(n=26)$, the authors were able to systematically compare the predictive power of the BSI and FACED scores for mortality. The core findings included: 1) both scoring systems yielded similar predictive power for mortality with high specificity; 2) a minor proportion of patients had discordant BSI and FACED scores (e.g. 11 patients with a severe BSI score actually had a mild FACED score); 3) compared with their counterparts, patients with severe BSI who had mild FACED score were younger and had better lung function, less extensive bronchiectasis, and nonsignificantly higher body mass index; and 4) based on the area under the receiver operating characteristic curve, the scoring systems did not differ statistically in the ability to predict mortality from a respiratory cause at 5, 10 and 15 years. Nonetheless, the FACED score had a greater predictive power for the 15-year mortality and a greater separation of the survival curves, indicating that the FACED score would be better suited to predicting survival on a longer term basis.

Admittedly, the BSI still harbours a complimentary role to the FACED score: the ability to predict the annual risk and outcomes of hospitalisation, which has not been fully addressed by the current study. What remains unresolved is the power of both scoring systems to predict future risks of bronchiectasis exacerbations and the differential responses to therapies (such as mucolytics [17] and inhaled antibiotics [18]). Furthermore, because of the relatively small sample size, the current study could not inform readers of the essential differences in future risks of bronchiectasis exacerbations, mortality or hospitalisation between the patients with high BSI scores who had low FACED scores and the patients with high BSI scores who had moderate-to-high FACED scores. Meanwhile, the usefulness of the BSI and FACED scores should be validated in independent cohorts such as the Oriental population, in which the prevalence of bronchiectasis might be significantly higher than that in Caucasians. Finally, because the BSI and FACED scores were derived from different cohorts, the components were assigned to varying weights; therefore, one may frequently encounter the problem of adding different weights to an identical clinical variable. Future efforts to further harmonise these two valuable composite scores in a larger population are needed.

Should there be novel scores other than the BSI and FACED for assessment of the disease severity? This remains an open question that needs to be adequately addressed in future cohort studies or large-scale clinical trials. It is anticipated that novel scores, if any, should also be sufficiently informative so that physicians might be able to readily determine the distinction in underlying pathophysiology (i.e. infection-, oxidative stress- or inflammation-predominant) or clinical phenotypes [19]. Because the currently available scores only included demographics, past history, lung function, symptoms and sputum bacteriology, there might still be room for the incorporation of other elements such as the clinically significant, well-validated biomarkers (i.e. C-reactive protein, interleukin-6 and matrix metalloproteinase). Another potential pitfall of the FACED score is that as a patient ages, there would be a concomitant increase in the apparent disease severity, albeit to a minor degree. How to exploit an ideal scoring system that is independent of demographics should also be taken into account in the future.

Finally, we come to an intriguing question: will the identification of bronchiectasis phenotypes provide additional benefits apart from the BSI or FACED scores? In our pilot study [19], FEV1, age of symptom 
onset, $P$. aeruginosa colonisation, frequency of hospitalisations and dyspnoea were associated with disease severity and future risks of exacerbations. Reassuringly, our findings are partially in agreement with the studies by Chalmers et al. [12] and Martinez-Garcia et al. [13]. Importantly, bronchiectasis patients might have a sizeable heterogeneity in clinical presentation despite their comparable disease severity (i.e. overlapped BSI scores in certain phenotypes), which justified the incorporation of clinical phenotyping apart from the disease severity assessment. Whilst this was a pilot study with small sample sizes $(n=148)$ and the current unsupervised clustering approach appears to be imperfect, the derivation of clinical phenotypes by using the international databases $[4,12,13]$ and the more comprehensive unsupervised learning approaches might continue to shed light on the nature of bronchiectasis, which will ultimately translate into personalised medical care.

The BSI and FACED score were not "born" alone, so their usefulness in predicting future risks of mortality may also be found together. Apart from the slight superiority in predicting 15-year mortality for the FACED score, current evidence coupled with the experience of clinical practice suggests the complimentary roles of both scoring systems. The extension of future work is expected to impart these scores with new elements that more comprehensively represent the genuine underlying pathophysiology of bronchiectasis; only then will we gain sufficient insights into the pathogenesis of bronchiectasis leading to significantly improved patient's healthcare.

\section{References}

1 Pasteur MC, Helliwell SM, Houghton SJ, et al. An investigation into causative factors in patients with bronchiectasis. Am J Respir Crit Care Med 2000; 162: 1277-1284.

2 Guan WJ, Gao YH, Xu G, et al. Aetiology of bronchiectasis in Guangzhou, southern China. Respirology 2015; 20: 739-748.

3 Chalmers JD, Aliberti S, Blasi F. Management of bronchiectasis in adults. Eur Respir J 2015; 45: 1446-1462.

4 Lonni S, Chalmers JD, Goeminne PC, et al. Etiology of non-cystic fibrosis bronchiectasis in adults and its correlation to disease severity. Ann Am Thorac Soc 2015; 12: 1764-1770.

5 Chalmers JD, Smith MP, McHugh BJ, et al. Short and long term antibiotic treatment reduces airway and systemic inflammation in non-cystic fibrosis bronchiectasis. Am J Respir Crit Care Med 2012; 7: 657-665.

6 Torrego A, Haque RA, Nguyen LT, et al. Capsaicin cough sensitivity in bronchiectasis. Thorax 2006; 61: 706-709.

7 Loukidies S, Horvath I, Wodehouse T, et al. Elevated levels of exhaled breath hydrogen peroxide in bronchiectasis. Am J Respir Crit Care Med 1998; 158: 991-994.

8 Pasteur MC, Bilton D, Hill AT, et al. British Thoracic Society guidelines for non-CF bronchiectasis. Thorax 2010; 65: Suppl. 1, i1-i58.

9 Restrepo M, Alborn JS. Bronchiectasis severity: Time to score. Am J Respir Crit Care Med 2014; 189: 508-509.

10 Reiff DB, Wells AU, Carr DH, et al. CT findings in bronchiectasis: limited value in distinguishing between idiopathic and specific types. Am J Radiol 1995; 165: 261-267.

11 Bhalla M, Turcios N, Aponte V, et al. Cystic fibrosis: scoring system with thin-section CT. Radiology 1991; 179: 783-788.

12 Chalmers JD, Goeminne P, Aliberti S, et al. The bronchiectasis severity index: an international derivation and validation study. Am J Respir Crit Care Med 2014; 189: 576-585.

13 Martinez-Garcia MA, de Gracia J, Vendrell Relat M, et al. Multidimensional approach to non-cystic fibrosis bronchiectasis: the FACED score. Eur Respir J 2014; 43: 1357-1367.

14 Saleh AD, Hurst JR. How to assess the severity of bronchiectasis? Eur Respir J 2014; 43: 1217-1219.

15 Minov J, Karadzinka-Bislimovska J, Vasilevska K, et al. Assessment of the non-cystic fibrosis bronchiectasis severity: the FACED score $v s$ the Bronchiectasis Severity Index. Open Respir Med J 2015; 9: 46-51.

16 Ellis HC, Cowman S, Fernandes M, et al. Predicting mortality in bronchiectasis using bronchiectasis severity index and FACED scores: a 19-year cohort study. Eur Respir J 2016; 47: 482-489.

17 Daviskas E, Anderson SD, Eberi S, et al. Effect of increasing doses of mannitol on mucus clearance in patients with bronchiectasis. Eur Respir J 2008; 31: 765-772.

18 Brodt AM, Stovold E, Zhang L. Inhaled antibiotics for stable non-cystic fibrosis bronchiectasis: a systematic review. Eur Respir J 2014; 44: 382-393.

19 Guan WJ, Jiang M, Gao YH, et al. Unsupervised learning technique identifies bronchiectasis phenotypes with distinct clinical characteristics. Int J Tubercul Lung Dis 2015 [In press DOI: 10.5588/ijtld.15.0500]. 